\title{
THE POLE OF ABDOMINAL DOPPLER ULTRASONOGRAPHY IN DIABETIC NEPHROPATHY DIAGNOSTICS
}

\author{
Liliya Stoyanova, Mariya Dimova, Elena Marinova, Anton Dinkov \\ Clinic of Internal Diseases, St. Marina University Hospital, Varna
}

\begin{abstract}
Diabetes mellitus is the most common chronic disorder of carbohydrate metabolism, which leads to progressive atherosclerosis and failure of multiple internal organs, which in turn results in an early and severe disability. Diabetic nephropathy is one of the main microvascular complications of this disease. The article explores the usefulness of abdominal Doppler ultrasonography to validate the early changes of renal hemodynamics in patients with diabetes mellitus.
\end{abstract}

Keywords: diabetes mellitus, diabetic nephropathy, abdominal Doppler ultrasonography, pulsed Doppler, resistivity Doppler index, pulsatility Doppler index

\section{INTRODUCTION}

Diabetes mellitus is the most common chronic disorder of carbohydrate metabolism. Its characteristic traits increased levels of blood sugar due to abnormal insulin secretion or function.

Diabetic nephropathy (DN) is one of the main microvascular complications of this disease. Diabetic glomerulosclerosis, a specific glomerulopathy, which develops during a long-term $\mathrm{DN}$ type 1 and 2, which in turn develops into different levels of proteinuria and a progressive damage of the kidney function. The syndrome was first described by Kimmelstiel and Wilson in 1936 after the introduction of insulin and the increase of the actual survival

Address for correspondence:

Liliya Stoyanova

St. Marina University Hospital

1 Hristo Smirnenski Blvd

9010 Varna

e-mail: lilstoianova67@abv.bg

Received: October 6, 2018

Accepted: December 17, 2018 rate of diabetics. In Bulgaria there are 550000 people with diabetes mellitus, $20-40 \%$ of which also have $\mathrm{DN}$. DN is the leading reason for end-stage renal disease (ESRD) (1).

Diabetes mellitus is a risk factor for cardiovascular disease. Around $20-25 \%$ of the patients with heart failure are diabetics $(2,3)$. Failure in the renal hemodynamics can lead to progressive deterioration of the heart function, to the so-called cardiorenal syndrome (CRS). It was described in 2004 and showed the complicated relationship between the kidneys and the heart.

Albuminuria is an early marker for renal dysfunction and a predictor of the presence of unwanted life-threatening events. That is why the early diagnostics of DN will decrease the risk of cardiovascular disease and mortality.

\section{Characteristics and Phases of DN}

The blood-urine barrier has three layers. It consists of the fenestrated endothelium of glomerular capillaries, basal membrane and podocytes (type of epithelial cells), which are using their foot processes to attach to the basal membrane. The foot processes from one podocyte intertwine with the foot process- 
es of the neighboring podocyte. In-between the foot processes are the so-called slit diaphragms. This barrier allows the filtration of the liquids from the capillaries to the Bowman's space by constraining the filtration of the big plasma molecules such as proteins (e.g., albumin) (4). The foot processes of the podocytes and the slit diaphragms are the thinnest strainer. Molecules with a relative weight of up to 100000 D can pass through them (Fig. 1) (5).

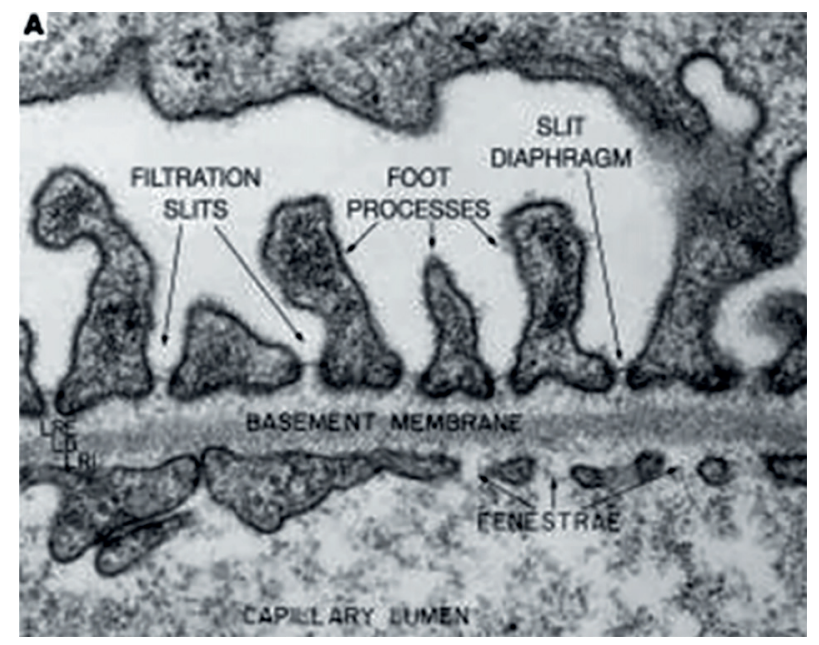

Fig. 1. Electron micrographs showing a peripheral region of a glomerular capillary where filtration takes place.The filtration surface consists of the endothelium, which is interrupted by fenestrae; the GBM; and the epithelial foot processes. The latter are attached to one another at their base by slit diaphragms. (Source: Farquhar MG. J Clin Invest. 2006; 116(8): 2090-3)

$\mathrm{DN}$ is a podocyte disease. It is known for having changes occurring in the foot processes of the podocytes and the slit diaphragms that can only be observed by electronic microscopy.

There are 5 phases of diabetic nephropathy:

1. Glomerular hyperfiltration without any morphological kidney changes, lack of microalbuminuria;

2. Early histological changes in the kidney, intermittent microalbuminuria;

3. Persistent microalbuminuria;

4. Proteinuria over $300 \mathrm{mg} / \mathrm{L}$;

5. ESRD.

DN diagnostics are needed not only because they prove the kidney engagement in the pathological process, but also because $\mathrm{DN}$ is associated with the occurrence of lethal cardiovascular events. It is known that patients with $\mathrm{DN}$ are rarely treated with kidney biopsy. It is needed in the cases where there is a presence of increasing proteinuria, short-term diabetes mellitus, lack of retinopathy. In all other cases the diagnosis is based on anamnestic and laboratory data (6). Diabetic kidney disease is observed in patients with diabetes mellitus of more than 10 years, bad glycemic control, presence of diabetic retinopathy, microalbuminuria, poor urinal sediment, sonographic results suggesting nephromegaly, thickened kidney parenchyma, increased echogenicity $1^{\text {st }}-2^{\text {nd }}$ degree. That is why the introduction in clinical practice of non-invasive and harmless methods could help early diagnostics of the disruptions of the renal hemodynamics for patients with diabetes mellitus.

Abdominal Doppler Ultrasonography - Characteristics and Application

Abdominal ultrasound is a first-choice method for examination of patients with diabetic nephropathy. It has many advantages compared to other instrumental methods. It is non-invasive, harmless and easily accessible. It can be applied on patients with different stages of renal diseases and it is not related to exposure to ionizing radiation. The introduction of Doppler techniques is taking the ultrasonography test to a higher level and makes it a reliable and informative method in angiology. The use of Doppler ultrasound and, more specifically, the test of the impedance indices provide us with the opportunity to detect changes in the peripheral vascular resistance.

The Resistivity index (RI) and the Pulsatility index (PI) show the vascular resistance and are dependent on both diastolic and systolic blood velocity (7).

Resistivity index /RI/= Peak systolic velocity (Vp) - End diastolic velocity (Vd)/Peak systolic velocity

Pulsatility index /PI/ = Peak systolic velocity (Vp) - End diastolic velocity (Vd)/Mean velocity

Here is what is considered to be normal values for each of the parameters above:

RI: 0.56 to $0.7 ; \mathrm{PI}: 0.7$ to 1.4 ; Vp: 30 to $140 \mathrm{~cm} /$ sec; Vd: 18 to $22 \mathrm{~cm} / \mathrm{sec}$.

Identifying RI has become a routine practice in diagnostics procedures. The examination is performed on an empty stomach. The patient should 
not have had any gas-forming food the night before. Curvilinear transducer operating at $3.5-4.0 \mathrm{MHz}$ is used. The right kidney is visualized by having the patient lie in a supine position. For the left kidney the patient must be in left side-up position. First, gray scale ultrasonography is done, followed by pulsedwave Doppler of the intrarenal artery. Finally, RI is measured. Three measurements are taken in the upper, mid and lower intrarenal arteries of each kidney. These three values are used to determine the average RI value (Fig. 2). During the measurement of RI the patient must hold his breath.

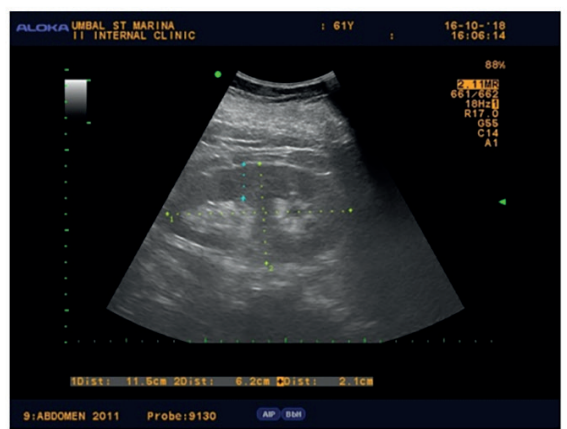

A.

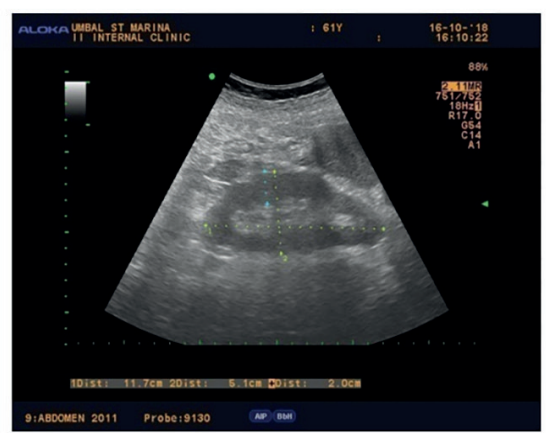

B. monitoring the chronic kidney disease. Nejad et al. studied 45 patients with type 2 diabetes mellitus and 30 healthy controls in 2009 (12). Doppler ultrasound was performed for all patients and controls, and RI was measured. The patients were categorized into 3 groups according to the severity of their nephropathy based on the serum creatinine level and 24-hour urine protein. They concluded that RI increased with the progression of $\mathrm{DN}$ and can be a predictive marker for renal impairment. Shaw et al. observed 60 patients with diabetes mellitus, type 2, between 2013 and 2015 (13). First, kidneys were visualized with

Fig. 2. A. 61-year old patient with long-term diabetes mellitus, type 2, with coronary artery disease, asthma attacks, hypertonic disease with good control of blood pressure and dyslipidemia; HbAlc-12.31\%, eGFR/MDRD/- $105 \mathrm{~mL} / \mathrm{min}$, albumin in a first morning fresh urine sample - $397 \mathrm{mg} / \mathrm{L}$, and albumin/creatinine ratio in a first morning fresh urine sample- $90.39 \mathrm{~g} / \mathrm{mol}$. A. and B. Kidneys, two-sided with upper-limit size, echogenicity of the parenchyma $1^{\text {st }}$ degree, thickness up to $2.1 \mathrm{~cm}$. C. Pulsed-wave Doppler-increased impedance indices - RI=0.83, PI=2.28

Nephromegaly and hyperfiltration are observed in the early stages of DN. In 2013, Mancini et al. reported that renal hypertrophy was present even in diabetic patients without proteinuria (9). Renal volume and RI values in patients with type 2 diabetes mellitus were significantly higher compared to the controls. The authors indicated the potential role of RI in the early identification of morphologic and hemodynamic renal changes in diabetic patients.

$\mathrm{RI}$ is a marker for endothelial dysfunction. High $\mathrm{RI}>0.73$ is associated with progression of $\mathrm{DN}(10)$.

Between 2011 and 2013, Shirin et al. tested 53 patients with diabetes mellitus, at an average age of $52.66 \pm 7.4$ years (29 males and 24 females) (11). They found out that RI was significantly higher for patients, who have developed nephropathy and correlated with the level of the serum creatinine and albumin in the urine. They recommend the use of RI in real-time B-mode scan and then intrarenal arteries were examined by duplex Doppler ultrasound. Thickness, echogenicity of the kidney parenchyma and RI of interlobar arteries were examined. With $18.2 \%$ of the patients it was proven that there was an increased echogenicity of the parenchyma of $1^{\text {st }}-2^{\text {nd }}$ degree. They also found out that RI had increased values even in patients with normal sonomorphology of the parenchyma and that it increased with the increase of the albuminuria. Ishimura et al. examined 112 patients with diabetes mellitus type 2 (65 men and 47 women, average age of 58 \pm 13 ) (14). They divided patients into four groups: group Iconsisted of patients with urinary albumin excretion (UAE) $<20 \mathrm{mcg} / \mathrm{min}(\mathrm{N}=42)$, group II with UAE $20-200$ $(\mathrm{N}=28)$, group III with UAE $>200(\mathrm{~N}=25)$, and group IV with serum creatinine $>1.5 \mathrm{mg} / \mathrm{dL}$ (N17) (Fig. 3, Fig. 4). 


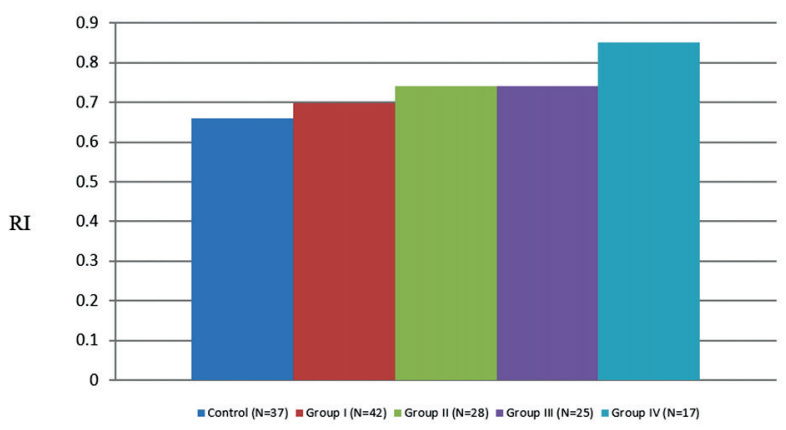

Fig. 3. Resistivity index (RI) of the interlobar artery of the control subjects and diabetic patients. Group I consisted of patients with urinaly albumin excretion (UAE) less than 20mcg/min (N=42); group II had UAE 20 and $<200 \mathrm{mcg} / \mathrm{min}(\mathrm{N}=28)$, group III had UAE $>200 \mathrm{mcg} / \mathrm{min}$ $(\mathrm{N}=25)$, and group IV had serum creatinine $>1.5 \mathrm{mg} / \mathrm{dL}$ $(N=17)$

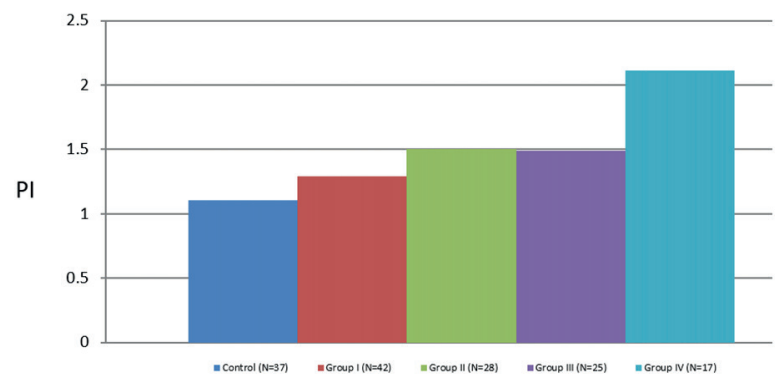

Fig. 4. Pulsatility index (PI) of the interlobar artety of the control subjectsand diabetic patients. Group divisions are the same as in Fig. 3

Ishimura et al.: Intrarenal vascular resistance in type II DM

The results showed significantly higher values of RI and PI where the diabetic nephropathy was at an advanced stage. They proved the positive correlation between RI and intima-media thickness (IMT) of the carotid and femoral arteries examined using B-mode echography. These results demonstrate that in the patients with diabetes mellitus type 2 , there are intrarenal hemodynamical dysfunctions, which are a result of the advancing atherosclerosis and endothelial dysfunction. In 2012, Youssef et al. tested 25 patients (15 females and 10 males) with T1-DM at a mean age of $10.8 \pm 2.2$ years and duration of diabetes of $5 \pm 1.1$ years (15). They reported a positive correlation between the increase of RI and age, duration of disease, and levels of glycated hemoglobin and GFR in children with T1-DM. The authors concluded that RI was increased early in TI-DM, and it could be a predictor of DN. In 2017, Genov et al. reported a sig- nificant increase of RI $(>0.7)$ in patients with diabetes mellitus, who were normotensive and with proteinuria (16). They discovered a negative correlation between RI and glomerular filtration, and a positive correlation between serum creatinine and blood urea nitrogen. In other researches, the authors point out an increase of RI with diabetics before the occurrence of microalbuminuria (17). They also considered RI as a marker for endothelial dysfunction and increased vascular resistance. It was concluded it could identify patients with a high risk of developing DN.

RI correlates with vascular resistance and the degree of the renal disease. It increases with all diffusing parenchymal processes in the kidney, which are observed not only in DN, but also in hypertension, gout, chronic glomerulopathies, collagenases, renal amyloidosis. However, the combination of nephromegaly, thickened renal parenchyma of over $2.0 \mathrm{~cm}$ with B-mode scan, increased RI and PI levels, known improper control of long-term diabetes mellitus, diabetic retinopathy and the lack of erythrocyturia are pointing out towards DN.

\section{CONCLUSION}

Abdominal Doppler ultrasonography is able to identify early dysfunctions of the renal hemodynamics inpatients with diabetic kidney disease. Impedance indices (RI and PI) should be included in the diagnostic algorithm of DN, together with the already established markers for renal dysfunction in the clinical practice such as serum creatinine, blood urea nitrogen, albuminuria, proteinuria and eGFR. RI and PI can be a useful tool for the doctor when it comes to prevention of micro- and macrovascular complications of diabetes mellitus.

\section{REFERENCES}

1. Kaludina I, Nenchev N. Renal complications of diabetes mellitus. Nephrology 2016:350-8. (in Bulgarian).

2. Georgiev B. Diabetes mellitus and heart failure. Science Cardiology. 2010;6:282-292.(in Bulgarian).

3. Georgiev B, Gocheva N, Tankova C. Diabetic cardiomyopathy mechanisms and therapy. Science Endocrinology.2008;2: 64-6.

4. Douglas E, Pooler J. Vander's Renal Physiology. $8^{\text {th }}$ edition. McGraw-Hill Medical;2011. p.7-9. 
Liliya Stoyanova, Mariya Dimova, Elena Marinova et al.

5. Galabov G, Vankov V. Human anatomy. Medicine and Physical Education;1986.p.325-32. (in Bulgarian).

6. Simeonov P.Diabetic glomerulosclerosis. Nephrology. Internal Medicine;336-8. (in Bulgarian).

7. Grigorov N.Abdominal Doppler ultrasonography. ZIP; 2010. p. 96-102. (in Bulgarian).

8. Nenov D. Nephrology handbook. Steno 2018.p. 4359. (in Bulgarian).

9. Mancini M, Masulli M, Liuzzi R, Mainenti RP, Ragucci M, Maurea S,et al.Renal duplex sonographic evaluation of type 2 diabetic patients. J Ultrasound Med;32(6):1033-40.doi: 10.7863/ultra.32.6.1033.

10. Masulli M, Mancini M, Liuzzi R, Daniele S, Mainenti PP, Vergara E,et al. Measurement of the intrarenal arterial resistance index for the identification and prediction of diabetic nephropathy. Nutr Metab Cardiovasc Dis. 2009;19(5):358-64.doi: 10.1016/j.numecd.2008.07.003.

11. Shirin M, Sharif MM, Gurung A, Datta A. Resistive index of intrarenal artery in evaluation of diabetic nephropathy. Bangladesh Med Res Counc Bull. 2015;41(3):125-30.

12. Nejad MN, Jafari B, Alipour P. Arterial Resistive index (RI) in type ii diabetic nephropathy stages and healthy controls. Iran J Radiol. 2009;6(1):29-32.
13. Shaw M, Bhagat $S$, Panda BB, Nisa $S$, Das B, Panda A. Diabetic nephropathy: Ultrasound, color doppler and biochemical correlation- a 2-year study. JMSCR.2016;4(8):12025-34. doi: 10.18535/jmscr/ v4i8.58.

14. Ishimura E, Nishizawa $Y$, Kawagishi T, Okuno Y, Kogawa K, Fukumoto S, et al. Intrarenal hemodynamic abnormalities in diabetic nephropathy measured by duplex Doppler sonography. Kidney Int. 1997;51(6):1920-74.

15. Youssef DM, Fawzy FM. Value of renal resistive index as an early marker of diabetic nephropathy in children with type-1 diabetes mellitus. Saudi J Kidney Dis Transpl.2012; 23(5):985-92. doi: 10.4103/1319-2442.100880.

16. Genov D, Kundurjiev A, Kostadinova A, Vutova C, Grozeva V. RI for the assessment of renal impairment in type 2 diabetes mellitus. National Nephrology Conference; 2017.

17. Thukral A,Mishra M, Srivastav V, Kumar H. Determinants of intravascular resistance in Indian diabetic nephropathy patients: a hospital-based study. Int J Vasc Med. 2011;2011:656030. doi: $10.1155 / 2011 / 656030$. 